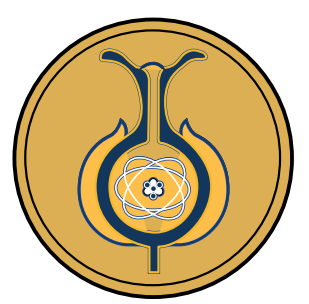

\title{
Estimación de parámetros genéticos de Gmelina arborea Roxb. (melina) en el Caribe de Costa Rica
}

Estimation of genetic parameters of Gmelina arborea Roxb. (melina) in the Costa Rican Caribbean

\author{
Estimativa de parâmetros genéticos da Gmelina arborea Roxb. (gamelina) no
} Caribe da Costa Rica

William Hernández-Castro ${ }^{1,2}$ - Yorleny Badilla Valverde ${ }^{3} \cdot$ Olman Murillo-Gamboa $^{3}$

Received: Jun/22/2020 • Accepted: Aug/31/2020 • Published: Jan/31/2021

\section{Resumen}

La Gmelina arborea es una de las especies de mayor importancia en proyectos de reforestación comercial en Costa Rica y actualmente, forma parte importante del programa de mejoramiento genético en Costa Rica liderado por GENFORES. El objetivo del presente estudio consistió en determinar los parámetros genéticos de 54 clones de melina utilizados en un ensayo clonal en la región Caribe de Costa Rica. El ensayo se estableció con un diseño de bloques completos al azar con cuatro repeticiones por tratamiento, con un distanciamiento de $4 \mathrm{~m} \times 3 \mathrm{~m}$. Se calcularon parámetros genéticos a los 10, 22 y 34 meses, para las variables diámetro a la altura del pecho (DAP), altura comercial, volumen comercial y calidad del fuste, utilizando el software SELEGEN, el cual se basa en el método REML/BLUP, además, se realizaron correlaciones genéticas entre las variables evaluadas. Los resultados muestran mayor heredabilidad individual en sentido amplio para las variables de volumen comercial y DAP, además, reflejan que la calidad del fuste a los 22 y 34 meses presenta los mayores coeficientes de heredabilidad individual y heredabilidad media del clon. Las correlaciones genéticas reflejan valores altos y positivos entre las variables DAP-volumen comercial y altura comercial-calidad del fuste, a los 34 meses de edad, con un 0.97 y 0.74 , respectivamente. Los clones 17 y 34 , mostraron ser los mejores en el ranking genético en términos de volumen comercial; el material de control y el clon 19, fueron los que se ubicaron en las últimas posiciones del ranking. Se obtuvo una ganancia genética a los 34 meses de edad de $6.11 \%$ y $9.36 \%$ en DAP y volumen comercial, respectivamente. Se concluye que existe variabilidad genética en los materiales genéticos utilizados, que permiten obtener ganancias genéticas importantes, sobre todo si se utilizan los primeros 10 clones del ranking genético de las accesiones evaluadas.

Palabras claves: mejoramiento genético; correlaciones genéticas; ganancia genética; parámetros genéticos.

\footnotetext{
William Hernández-Castro, $\triangle$ william.hernandez.castro@una.cr, (10 https://orcid.org/0000-0003-2416-8329

Yorleny Badilla Valverde, $\square$ yorlenybadilla@yahoo.es, (1) https://orcid.org/0000-0002-6743-9734

Olman Murillo-Gamboa, $\triangle$ olmuga@yahoo.es, (D) https://orcid.org/0000-0003-3213-8867

1 Instituto de Investigación y Servicios Forestales, Universidad Nacional, Heredia, Costa Rica.

2 Doctorado en Ciencias Naturales para el Desarrollo DOCINADE, Heredia, Costa Rica.

3 Escuela de Ingeniería Forestal, Instituto Tecnológico de Costa Rica, Cartago, Costa Rica.
} 


\section{Abstract}

Gmelina arborea is one of the most important species in commercial reforestation projects in Costa Rica and is currently a significant part of the genetic improvement program led nationally by GENFORES. The objective of the present study is to determine the genetic parameters of 54 melina clones used in a clonal trial in the Caribbean region of Costa Rica. The trial was established in a randomized complete block design with four replications per treatment, with a distance of $4 \mathrm{~m} \times 3 \mathrm{~m}$. Genetic parameters were calculated at 10,22 , and 34 months, for the variables diameter at breast height (DBH), commercial height, commercial volume, and stem quality, using the SELEGEN REML/BLU software. Genetic correlations were also made between the evaluated variables. Results indicated greater individual heritability in the broad sense for the commercial volume and DBH variables. In addition, the quality of the stem at 22 and 34 months presents the highest coefficients of individual heritability and the average heritability of the clone. The genetic correlations reflect high and positive values between the DBH-commercial volume-commercial heightstem quality variables, at 34 months of age, with 0.97 and 0.74 , respectively. Clones 17 and 34 showed to be the best in the genetic ranking in terms of commercial volume, while the material control and clone 19 were last in the ranking. A $6.11 \%$ and $9.36 \%$ genetic gain was obtained at 34 months of age in DBH and commercial volume, respectively. It is concluded that there is genetic variability in the materials used, which allows us to obtain significant genetic gain, especially if using the first 10 clones of the genetic ranking.

Keywords: genetic improvement; genetic correlations; genetic gain; genetic parameters

\section{Resumo}

A Gmelina arborea é uma das espécies de maior importância em projetos de reflorestamento comercial na Costa Rica e, atualmente, forma parte importante do programa de melhoramento genético na Costa Rica liderado pela GENFORES. 0 objetivo do presente estudo consistiu em determinar os parâmetros genéticos de 54 clones de gamelina utilizados em um ensaio clonal na região do Caribe na Costa Rica. 0 ensaio foi estabelecido com um desenho de blocos completos aleatórios com quatro repetições por tratamento, com uma distância de $4 \mathrm{~m} \times 3 \mathrm{~m}$. Foram calculados parâmetros genéticos aos 10, 22 e 34 meses, para as variáveis diâmetro à altura do peito (DAP), altura comercial, volume comercial e qualidade do fuste, utilizando 0 software SELEGEN, que se baseia no método REML/BLUP, além disso, foram realizadas correlações genéticas entre as variáveis avaliadas. Os resultados apresentam maior herdabilidade individual no sentido amplo para as variáveis de volume comercial e DAP; também, mostram que a qualidade do fuste aos 22 e 34 meses apresenta os maiores coeficientes de herdabilidade individual e herdabilidade média do clone. As correlações genéticas mostram valores altos e positivos entre as variáveis DAP-volume comercial e altura comercial-qualidade do fuste, aos 34 meses de idade, com 0,97 e 0,74, respectivamente. Os clones 17 e 34 , mostraram ser os melhores no ranking genético em termos de volume comercial; enquanto o material de controle e o clone 19 foram os que se localizaram nas últimas posições do ranking. Obteve-se um ganho genético aos 34 meses de idade de 6,11\% e 9,36 \% em DAP e volume comercial, respectivamente. Concluise que existe variabilidade genética nos materiais genéticos utilizados, permitindo a obtenção de ganhos genéticos importantes, especialmente se forem utilizados os primeiros 10 clones do ranking genético dos acessos avaliados.

Palavras-chave: melhoramento genético; correlações genéticas; ganho genético; parâmetros genéticos. 


\section{INTRODUCCIÓN}

La reforestación comercial en Costa Rica se basa principalmente, en la utilización de dos especies forestales, donde la Gmelina arborea (melina) se consolidó como una de las principales especies a plantar en Costa Rica (Moya, 2004; Murillo y Guevara, 2013), alcanzando 118235 hectáreas de área plantada y 126470 árboles plantados en sistemas agroforestales o de manera dispersa (INEC, 2014).

En la actualidad, la madera de melina sigue posicionada en el mercado local, por su versatilidad, es utilizada para la fabricación de material de embalaje, para la industria de construcción y para la ebanistería (Barrantes y Ugalde, 2019; Moya, 2004). Es la segunda especie en importancia, en términos de volumen aprovechable (103 $362 \mathrm{~m}^{3} / \mathrm{año)} \mathrm{y} \mathrm{valor} \mathrm{económico} \mathrm{de} \mathrm{la}$ madera en pie, en patio y aserradero, con montos de 2993, 5837 y 20343 millones de colones por año (Chavarría, 2016; Barrantes y Ugalde, 2019).

Con el impulso de los programas de mejoramiento en Costa Rica, a partir de la década los años 90 (Murillo, 1992; Zeaser, 1998), se inicia un proceso que se consolida bajo una estrategia de mejoramiento genético, basada en la silvicultura clonal (Badilla y Murillo, 2011). A partir del año 2002, se continua con este programa, liderado por la Cooperativa Genética Forestal (GENFORES), para varias especies forestales dentro de las cuales se encuentra Gmelina arbórea.

Este programa generó un paquete tecnológico que permitió abordar los proyectos de reforestación con la especie melina y esto de manera integral, al tomar en cuenta aspectos relacionados con el incremento del crecimiento, la productividad, las propiedades físico-mecánicas, la resistencia o la tolerancia a enfermedades, la calidad de la madera, y la forma del fuste, entre otros.

La evolución que ha experimentado el sector forestal en las últimas décadas y la aplicación de herramientas como la silvicultura clonal y la selección genética han potenciado un aumento en la productividad de las plantaciones de Gmelina arborea. En el pacífico sur de Costa Rica, la selección de árboles plus de melina registraron un diferencial de selección sobre la población base, de 19.8; 54, y 54.4, para el DAP, volumen y calidad del fuste, respectivamente, (Badilla y Murillo, 2011; Balcorta y Vargas, 2004), reduciendo significativamente, los turnos de corta a 5-6 años, con la consecuente producción de madera para diversos usos (Ávila, et ál., 2015).

La estimación de los parámetros genéticos, tales como el coeficiente de variación, la heredabilidad y las correlaciones, son de utilidad en el establecimiento de programas de mejoramiento genético, ya que permiten direccionar la selección de individuos con características superiores (Vencovsky \& Barriga, 1992; Zimback et al., 2011; Zobel y Talbert, 1984).

En este sentido el programa de mejoramiento genético con Gmelina arborea en Costa Rica, cuenta con una amplia base, cuyos conjuntos genéticos se han venido evaluando en diferentes condiciones de clima, suelo y manejo silvicultural, con el propósito de incrementar la productividad y la calidad de la materia prima generada.

De acuerdo con Kumar (2007), los parámetros genéticos son herramientas útiles para predecir la ganancia que se espera del material clonal. La variación entre clones se usa comúnmente como una estimación de la variación genética total y para calcular el grado de control, para un carácter particular (Foster y Shaw, 1988). La estimación 
precisa de parámetros es indispensable en su mejoramiento. Para la de los componentes de la variancia es importante que los individuos sean originados a partir de una población de base amplia, por lo tanto, se debe conocer el grado de parentesco entre los materiales evaluados.

La varianza genética aditiva se destaca entre los parámetros estimados, por ser el principal indicador de las propiedades genéticas en una población y responsable de promover cambios en el carácter sometido a procesos de selección, dado que el efecto aditivo de los genes controla la mayoría de los caracteres cuantitativos de importancia económica (Falconer, 1987).

La estimación de parámetros genéticos permite obtener información sobre la naturaleza de la acción genética en la herencia de los caracteres, al permitir la base para la evaluación requerida por el mejoramiento, además de ofrecer información esencial para la selección y definición de programas de mejoramiento de una población (Marques-Junior, 1995).

Estas estimaciones son necesarias cuando se desea predecir ganancias genéticas o evaluar la viabilidad de un programa de mejoramiento, así como también, apoyar de forma efectiva el progreso genético en dichos programas. Dentro de los parámetros más importantes se destacan los coeficientes de variación genética y la heredabilidad, las correlaciones genéticas y fenotípicas entre caracteres y la precisión de la selección (Cruz, 2005). Por lo tanto, el objetivo del presente trabajo fue determinar los parámetros genéticos de materiales superiores de Gmelina arbórea, para darle continuidad a los programas de mejoramiento forestal de la especie.

\section{METODOLOGÍA}

Área de estudio: el trabajo se realizó con información proveniente de un ensayo genético de clones de Gmelina arborea, establecido durante el mes de junio del 2015, en Cariari de Pococí, provincia de Limón, en las coordenadas geográficas $\mathrm{N}$ $10^{\circ} 44^{\prime} 82^{\prime \prime}$ y W $-83^{\circ} 79^{\prime} 06^{\prime}$ ", con el objetivo de generar información sobre el comportamiento de los clones para el programa de mejoramiento genético de Gmelina arborea de GENFORES.

El ensayo fue instalado con un diseño experimental en bloques completos al azar, con cuatro repeticiones por tratamiento (clones). Los clones son distribuidos aleatoriamente, en parejas dentro de cada bloque, con la condición de que no queden juntas un mismo tratamiento (parejas) dentro de un mismo bloque. El material genético pertenece a la empresa BARCA, y se utilizaron 54 clones y un testigo, los cuales se plantaron a una densidad de 833 árboles por hectárea, con distanciamiento de cuatro metros entre hileras y tres metros entre árboles.

Descripción del área de estudio: la topografía del terreno es casi plana con una pendiente menor al $5 \%$, en general, no presenta problemas de inundación o encharcamiento, por poseer una red de drenajes para la canalización del exceso de agua por lluvias, ausencia de compactación del suelo, sin embargo, previo al establecimiento del ensayo se realizó una preparación mecánica del área del terreno, además, se realizó una aplicación de fertilizante químico (10-30-10) con una dosis de 50 gramos por planta, en el momento de la siembra, con el propósito de favorecer el desarrollo radicular de las plantas.

El tipo de suelo se clasifica dentro del orden Inceptisol (Mata-Chinchilla y 
Castro-Chinchilla, 2019) y el área de estudio se ubica dentro de la zona de vida Bosque muy Húmedo Tropical; presenta una temperatura media anual entre $26^{\circ} \mathrm{C}-28^{\circ} \mathrm{C}$ y una precipitación media anual entre 4000 $\mathrm{mm}$ - $5000 \mathrm{~mm}$, con una evapotranspiración media anual mayor a $1100 \mathrm{~mm}$ y menor a $1200 \mathrm{~mm}$, un brillo solar en promedio anual de $4-5$ horas por día (IMN, 2005).

Recolección y análisis de la información: el ensayo clonal se midió en tres ocasiones, a los 10 meses, a los 22 meses y a los 34 meses. Para el levantamiento de la información se utilizó hojas de campo y el respectivo croquis que mostraba el diseño del ensayo. Se midieron las variables diámetro a la altura de pecho (DAP), altura comercial y la calidad del fuste comercial, que se cuantifica con base en la calidad de las primeras cuatro trozas, con una longitud de 2.5 metros cada una, donde 1 es la máxima calidad para aserrío y 4 es una troza sin valor comercial o utilizable para leña.

En el formulario de campo, se registró el lugar de experimento, bloque, número de fila dentro de cada uno, código de clon, testigo y número dentro de la pareja de árboles por evaluar ( 1 o 2), así como los datos correspondientes a las diferentes variables por evaluar.

Análisis de datos: para el análisis de la información se procedió a elaborar una base de datos que integrara las diferentes mediciones de las variables evaluadas a cada clon, en cada uno de los bloques del ensayo. Posteriormente, a partir de los datos de DAP y altura comercial se procedió a calcular el volumen comercial con corteza, utilizando la siguiente ecuación (1):

$$
\mathrm{Vc}=\left((D A P / 100)^{2} *(\pi / 4) *(\text { Hcom })\right) * 0.65
$$

Donde $\mathrm{Vc}=$ Volumen comercial con corteza $\mathrm{DAP}=$ Diámetro a la altura del pecho, medido a $1.3 \mathrm{~m}$ en cada árbol, con cinta diámetrica.

Hcom=Altura comercial estimada a partir de la cantidad de trozas comerciales posibles de $2.5 \mathrm{~m}$ largo en cada árbol.

Para el cálculo de la calidad del fuste se tomó en cuenta la cantidad de trozas estimadas por individuo y el valor asignado, según la calificación para cada troza, para esto se utilizó la metodología para la valoración de plantaciones forestales desarrollada por Murillo et al., (2004). La ecuación 2 permite calcular la calidad global del árbol, tomando en cuenta el aporte individual de cada troza; en ese caso se corresponde a un árbol cuyo fuste cuenta con cuatro trozas.

$$
\begin{gathered}
\text { Calidad general }=T 1 * 0.40+T 2 * 0.30+ \\
T 3 * 0.20+T 4 * 0.10
\end{gathered}
$$

Donde: $T 1, T 2, T 3$ y $T 4$ : calidades (en escala de 1 a 4 ) asignadas a cada toza.

En vista de que la calidad general del árbol oscila entre 1 y 4 , se procedió a transformar la calidad en una escala de 1 a 100, para lo cual se utilizó el algoritmo descrito por la ecuación 3 :

Calidad $(\%)=100 *\left[1-\left(\frac{\text { calidad general }-1}{3}\right)\right]$

Posteriormente, se procedió a conformar una base de datos en Excel que unificara para cada clon dentro de cada bloque todas las variables evaluadas y para cada una de las mediciones realizadas para su respectivo procesamiento.

Los análisis se realizaron utilizando el software SELEGEN, el cual se basa en el procedimiento estadístico REML/BLUP 
(Resende, 2007), utilizando el modelo 2, el cual se usa en ensayos dispuestos en bloques completos al azar, clones no emparentados, varias plantas por parcela, y el modelo estadístico se presenta en la ecuación 4.

$$
Y=X r+Z a+W p+T i+e
$$

Donde, $Y$ es el vector de los datos y es un parámetro común entre todas las familias evaluadas, $r$ corresponde a los efectos de la repetición (que se asumen como fijos) sumados a la media general, $a$ corresponde a los efectos genéticos aditivos característicos (que se asumen como aleatorios), $p$ corresponde a los efectos de parcela (asumidos como aleatorios), $i$ corresponde a los efectos de la interacción genotipo $\mathrm{x}$ ambiente (aleatorios) y $e$ corresponde a los efectos del error experimental (aleatorios). Las letras mayúsculas representan las matrices de incidencia para los efectos referidos (Resende, 2007).

Dicho modelo permite estimar la varianza genética $(\mathrm{Vg})$, varianza ambiental entre parcela (Vparc), varianza residual o no explicada por el modelo (Ve), varianza fenotípica total (Vf), heredabilidad individual en sentido amplio $\left(\mathrm{H}^{2} \mathrm{~g}\right)$, coeficiente de determinación de la parcela ( $\mathrm{C}^{2}$ parc), heredabilidad media del clon $\left(\mathrm{H}^{2} \mathrm{mc}\right)$, precisión en la estimación de los parámetros (Acclonal), coeficiente de variación genética, coeficiente de variación experimental (CVe \%) $\mathrm{y}$ el coeficiente de variación relativa $(\mathrm{CVr})$ (Resende, 2007).

Posteriormente, se realizaron correlaciones genéticas (r) que permitan analizar interrelaciones entre las diversas variables evaluadas, a través del modelo estadístico 102 del software SELEGEN (Resende, 2007). Una vez estimados los coeficientes de correlación se confirmó la significancia estadística para " $r$ ", mediante una prueba T, dada por la siguiente formula:

$$
\mathrm{Tc}=[\mathrm{r} \sqrt{n-2}] /\left[\sqrt{1-r^{2}}\right]
$$

La " $T$ " calculada (Tc) se comparó con una $\mathrm{T}$ de la tabla $(\mathrm{Tt})$, al nivel de significancia de 0.05 y 0.01 , y con (n-2) grados de libertad.

\section{RESULTADOS}

El ensayo clonal acusó un crecimiento uniforme, tanto en DAP como en altura comercial, sobre todo a partir de la segunda evaluación, además, de una buena calidad del fuste. Durante los primeros 10 meses del ensayo la tasa de crecimiento de las variables fue menor con relación en los períodos posteriores de evaluación, donde el DAP fue la variable que mostró mayor crecimiento, pasando de $6.69 \mathrm{~cm}$ a los 10 meses, hasta llegar a $21 \mathrm{~cm}$ a los 34 meses de edad, presentando un valor máximo de $28.3 \mathrm{~cm}$ y un valor mínimo de $8.7 \mathrm{~cm}$, a los 34 meses (cuadro 1).

La altura comercial presentó un comportamiento menos diferenciado durante los periodos de evaluación con un valor de 4.73 m, $9.08 \mathrm{~m}$ y $9.63 \mathrm{~m}$, para el primero, segundo y tercer período de medición, respectivamente, con un rango de valores entre 2.5 $\mathrm{m}$ y $10 \mathrm{~m}$ de altura comercial. El volumen comercial, al ser una variable que involucra tanto el DAP como la altura comercial para su cálculo, está influenciado por ambas, mostrando un crecimiento de 0.0112 $\mathrm{m}^{3}, 0.1418 \mathrm{~m}^{3}$ y $0.2247 \mathrm{~m}^{3}$, a los 10,22 y 34 meses de edad, respectivamente (cuadro 1).

La calidad del fuste presentó valores promedio de $89 \%$ y $84 \%$, a los 22 y 34 meses de edad, respectivamente, con un valor máximo de $100 \%$ en ambas edades y 
un valor mínimo de $20 \%$ y $33 \%$, respectivamente, según las edades de medición. Con relación en el coeficiente de variación, se muestra una disminución de los valores conforme aumenta el periodo de evaluación, a excepción de la calidad del fuste, donde se presenta un leve aumento en dicho coeficiente (Cuadro 1).

Los parámetros calculados a excepción de la altura comercial (AltCom), mostraron un comportamiento ascendente de acuerdo con la edad de los clones. La calidad del fuste fue la variable que presentó el mayor valor de heredabilidad individual, con valores de $\mathrm{h}^{2} \mathrm{~g}=0.10$ y 0.15 a la edad de 10 y 22 meses. Con relación en el DAP, altura comercial y volumen comercial, a pesar de mostrar diferencias en el coeficiente de heredabilidad individual, en promedio muestran un valor similar, igual $\mathrm{a} \mathrm{h}^{2} \mathrm{~g}=0.07$. En términos de heredabilidad media del clon, el DAP presentó los mayores valores, con un promedio de $h^{2} \mathrm{mc}=0.74$, seguido por el volumen comercial, el cual presentó un valor de $h^{2} \mathrm{mc}=0.70$; la altura comercial mostró un valor de $\mathrm{h}^{2} \mathrm{mc}=0.63$. Con relación en la calidad del fuste, a los 22 y 34 meses de edad presentó un coeficiente de heredabilidad media del clon en promedio de 0.83 (cuadro 2).

El coeficiente de variación genética y variación ambiental es bajo, donde el volumen comercial es la variable que presenta mayores valores en ambos coeficientes,

Cuadro 1. Características dasométricas de las variables de crecimiento de ensayo clonal de Gmelina arborea (melina) en Cariari de Pococí, provincia de Limón, Costa Rica.

\begin{tabular}{lcccccccccccc}
\hline \multicolumn{1}{c}{ Variable / Edad (meses) } & \multicolumn{3}{c}{ DAP (cm) } & \multicolumn{3}{c}{ AltCom (m) } & \multicolumn{3}{c}{ VolCom $\left(\mathbf{m}^{\mathbf{3}}\right)$} & \multicolumn{3}{c}{ Calidad del fuste (\%) } \\
& $\mathbf{1 0}$ & $\mathbf{2 2}$ & $\mathbf{3 4}$ & $\mathbf{1 0}$ & $\mathbf{2 2}$ & $\mathbf{3 4}$ & $\mathbf{1 0}$ & $\mathbf{2 2}$ & $\mathbf{3 4}$ & $\mathbf{1 0}$ & $\mathbf{2 2}$ & $\mathbf{3 4}$ \\
\hline Promedio & 6.69 & 17.03 & 21 & 4.17 & 9.08 & 9.63 & 0.0112 & 0.1418 & 0.2247 & - & 89 & 84 \\
Máximo & 12.9 & 23.30 & 28.3 & 6.42 & 10 & 10 & 0.0483 & 0.2701 & 0.4089 & - & 100 & 100 \\
Mínimo & 2 & 7.8 & 8.7 & 1.9 & 2.5 & 2.5 & 0.0004 & 0.0078 & 0.0097 & - & 20 & 33 \\
Desviación estándar & 1.91 & 2.33 & 2.83 & 0.77 & 1.59 & 1.17 & 0.01 & 0.05 & 0.07 & - & 16.96 & 16.58 \\
Coeficiente de variación (\%) & 28.56 & 13.7 & 13.51 & 18.50 & 17.50 & 12.1 & 66.69 & 35.72 & 31.15 & - & 18.98 & 19.81 \\
\hline
\end{tabular}

Nota: AltCom: altura comercial / VolCom: volumen comercial.

Cuadro 2. Estimación de parámetros genéticos de un ensayo clonal de Gmelina arborea en tres edades diferentes, para los caracteres de DAP, altura comercial (AltCom), volumen comercial (VolCom) y calidad del fuste, en Cariari de Pococí, provincia de Limón, Costa Rica.

\begin{tabular}{lccccccccccc}
\hline \multicolumn{1}{c}{$\begin{array}{c}\text { Parámetros } \\
\text { genéticos }\end{array}$} & \multicolumn{1}{c}{} & DAP & \multicolumn{4}{c}{ Caracteres y valores por edad de medición } & \multicolumn{3}{c}{ VolCom } & \multicolumn{2}{c}{ Calidad } \\
& $\mathbf{1 0}$ & $\mathbf{2 2}$ & $\mathbf{3 4}$ & $\mathbf{1 0}$ & $\mathbf{2 2}$ & $\mathbf{3 4}$ & $\mathbf{1 0}$ & $\mathbf{2 2}$ & $\mathbf{3 4}$ & $\mathbf{2 2}$ & $\mathbf{3 4}$ \\
\hline $\mathbf{H}^{2} \mathbf{g}$ & 0.06 & 0.07 & 0.07 & 0.08 & 0.06 & 0.06 & 0.05 & 0.07 & 0.08 & 0.10 & 0.15 \\
$\mathbf{H}^{2} \mathbf{m c}$ & 0.66 & 0.77 & 0.78 & 0.61 & 0.56 & 0.71 & 0.59 & 0.72 & 0.79 & 0.80 & 0.87 \\
$\mathbf{C V g} \%$ & 7.55 & 3.51 & 3.55 & 5.06 & 4.59 & 2.92 & 14.40 & 8.85 & 8.04 & 6.14 & 7.34 \\
CVe \% & 13.31 & 4.76 & 4.62 & 10.00 & 8.96 & 4.63 & 29.16 & 13.41 & 10.16 & 7.52 & 6.84 \\
CVr \% & 0.57 & 0.74 & 0.77 & 0.51 & 0.46 & 0.63 & 0.49 & 0.66 & 0.79 & 0.82 & 1.07 \\
Precisión & 0.81 & 0.88 & 0.88 & 0.78 & 0.75 & 0.84 & 0.77 & 0.85 & 0.89 & 0.89 & 0.93 \\
GG \% & 8.10 & 4.64 & 6.11 & 4.09 & 3.50 & 2.15 & 14.60 & 10.23 & 9.36 & 6.00 & 5.18 \\
\hline
\end{tabular}

Nota: $h^{2} g$ es la heredabilidad individual, $h^{2} m c$ es la heredabilidad media entre clones, $\mathrm{CVg}$ es el coeficiente de variación genético, $\mathrm{CVe}$ es el coeficiente de variación ambiental, $\mathrm{CVr}$ es la relación entre el coeficiente de variación genética y el coeficiente de variación ambiental y GG es la ganancia genética. 
mientras que los mayores valores de coeficientes de variación relativa $(\mathrm{CVr})$ lo presentan el DAP, el volumen comercial y la calidad del fuste, a los 34 meses de edad. Para el caso del coeficiente de variación genético, el mayor valor se presenta a los 10 meses, con $14.4 \%$ en volumen comercial, el cual va disminuyendo conforme la edad, este mismo comportamiento se presenta con los otros caracteres evaluados y para los coeficientes de variación ambiental (cuadro 2).

Para el caso del coeficiente de valor relativo, es a la inversa, conforme aumenta la edad este valor tiende subir. Los parámetros genéticos estimados para las distintas variables se pueden considerar confiables en la mayoría de los casos y para las tres evaluaciones realizadas, en vista de que todos presentan valores iguales o superiores a $75 \%$ de precisión (cuadro 2 ).

En términos de ganancia genética la tendencia de los valores obtenidos por carácter es descendente conforme aumenta la edad. El volumen comercial presenta los mayores valores de ganancia genética durante el período de evaluación, seguido del DAP. La altura comercial y la calidad del fuste presentan los valores más bajos, respectivamente. A la edad de 34 meses lo valores para el DAP, volumen comercial, altura comercial y calidad del fuste es de $6.11 \%, 9.36 \%, 2.15 \%$ y $5.18 \%$, respectivamente (cuadro 2 ).

El valor genético del volumen comercial permite conocer los límites inferior y superior para cada uno de los genotipos evaluados y a la vez compararlo con el valor promedio obtenido para la variable del volumen comercial a determinada edad. Específicamente, a la edad de 34 meses este valor genético se encuentra entre un límite inferior dentro del rango de $0.1532 \mathrm{~m}^{3}$ a $0.2325 \mathrm{~m}^{3}$ y uno superior que se encuentra desde los $0.1869 \mathrm{~m}^{3}$ a $0.2740 \mathrm{~m}^{3}$ (figura 1). Lo anterior, comparado con el promedio del volumen comercial del ensayo a la misma edad (34 meses), el cual corresponde a 0.2247 (cuadro 1) y permite conocer cuáles clones se encuentran por encima o por debajo de la media obtenida.

La figura 1 permite observar gráficamente, el valor genético del volumen comercial, de acuerdo con los elementos descritos anteriormente, además, permite observar cuales son los mejores clones de acuerdo con la posición en el ranking genético, donde los clones 17 y 34 , ocupan los dos primeros lugares, mientras que el material testigo

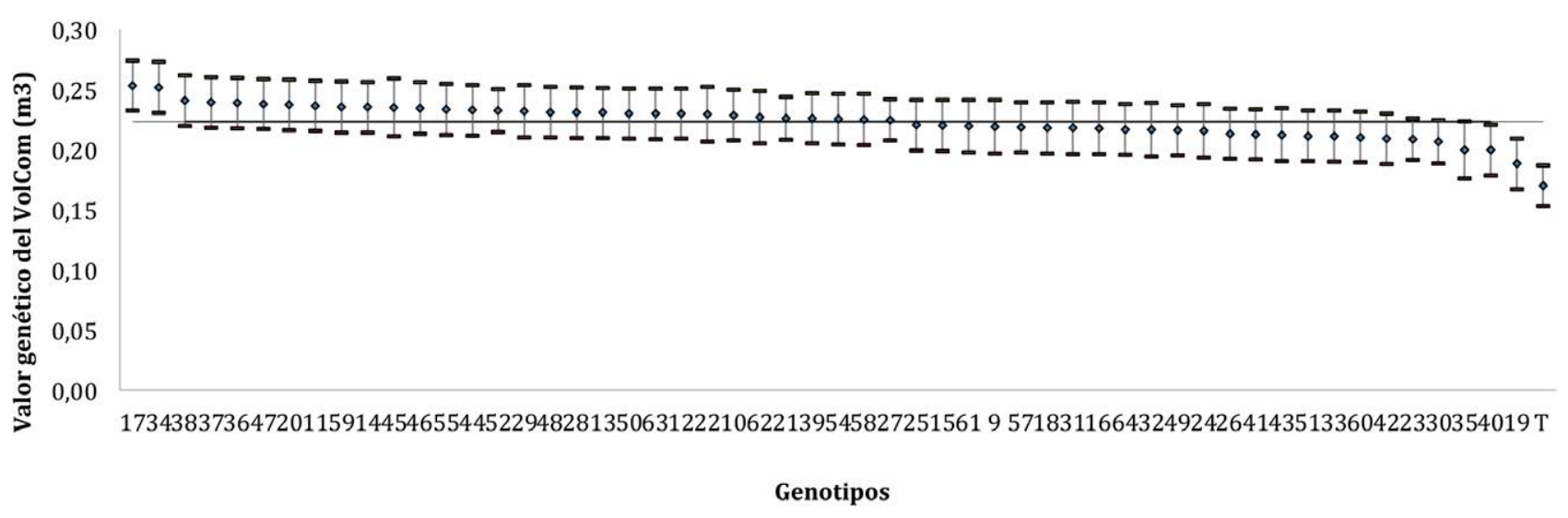

Figura 1. Valores genéticos para la variable volumen comercial $\left(\mathrm{m}^{3}\right)$ estimado a los 34 meses de edad para 55 genotipos en Cariari de Pococí, provincia de Limón, Costa Rica. 
y el clon 19 , son los que se localizan en la última y penúltima posición del ranking, a la vez se puede observar que poco menos de la mitad de los clones (26), se ubican por encima del promedio general y constituyen los individuos más productivos en términos de volumen comercial el ensayo genético.

Los mayores valores de correlaciones genéticas se presentaron entre el DAP y el volumen comercial, en las tres evaluaciones realizadas. Al correlacionar el DAP a los 22 meses con el volumen comercial a los 22 y 34 meses, se obtuvo valores de 0.90 y 0.80 , los cuales reflejaron correlaciones estadísticamente significativas, posteriormente, a los 34 meses la correlación entre el DAP y el volumen comercial aumenta significativamente, alcanzando un valor de 0.97 , estadísticamente, significativo. Además, a la edad de 22 meses se presentan correlaciones con valores significativos, de 0.73 y 0.63 entre la altura comercial y la variable calidad del fuste, que aumenta al correlacionar dichas variables a los 34 meses con un valor de 0.74. (Cuadro 3).

El ranking genético permite conocer la posición de los clones de acuerdo con su rendimiento, en este caso, en términos de volumen comercial. Los clones 34 y 17 se mantienen estables durante todo el período de evaluación, los 36, 52, 38, 47 y 14, mostraron una tendencia ascendente, mientras que otros como el 28, 51, 21 y el 60 mostraron un descenso en el ranking genético. Los clones 19, 40, 42, 35 y el testigo se ubicaron siempre en los últimos lugares del ranking genético (figura 2).

\section{Discusión}

Los resultados obtenidos para las variables dasométricas reflejan un crecimiento que puede ser catalogado como moderado a bueno, comparado con otros datos de crecimiento registrados en región Atlántica de Costa Rica, donde se obtuvo

Cuadro 3. Correlación genética entre las edades de medición para los caracteres de DAP, altura comercial, volumen comercial y calidad del fuste, de un ensayo clonal de Gmelina arborea, en Cariari de Pococí, provincia de Limón, Costa Rica.

\begin{tabular}{|c|c|c|c|c|c|c|c|c|c|c|c|}
\hline \multirow{2}{*}{$\begin{array}{c}\text { Edad } \\
\text { Variable }\end{array}$} & \multicolumn{3}{|c|}{10 meses } & \multicolumn{4}{|c|}{22 meses } & \multicolumn{4}{|c|}{34 meses } \\
\hline & DAP & AltCom & VolCom & DAP & AltCom & VolCom & CalFuste & DAP & AltCom & VolCom & CalFuste \\
\hline DAP & - & $0.85 * *$ & $0.97 * *$ & 0.36 & 0.22 & 0.35 & 0.18 & $0.51^{*}$ & 0.13 & $0.47^{*}$ & -0.01 \\
\hline AltCom & & - & $0.86^{* *}$ & $0.46^{*}$ & 0.24 & $0.42 *$ & 0.06 & $0.62 *$ & 0.14 & $0.56^{*}$ & -0.04 \\
\hline VolCom & & & - & $0.39^{*}$ & 0.22 & $0.38^{*}$ & 0.15 & $0.53 *$ & 0.13 & $0.49^{*}$ & 0.02 \\
\hline DAP & & & & - & $0.39^{*}$ & $0.90 * *$ & 0.004 & $0.83 * *$ & $0.40^{*}$ & $0.80^{*}$ & 0.20 \\
\hline AltCom & & & & & - & $0.73 *$ & $0.73 *$ & 0.50 & $0.64 *$ & $0.58^{*}$ & $0.63^{*}$ \\
\hline VolCom & & & & & & - & 0.36 & $0.82 *$ & $0.58^{*}$ & $0.84 * *$ & $0.45^{*}$ \\
\hline Calidad & & & & & & & - & 0.10 & $0.55^{*}$ & 0.24 & $0.77 *$ \\
\hline DAP & & & & & & & & - & $0.50^{*}$ & $0.97 * *$ & 0.24 \\
\hline AltCom & & & & & & & & & - & $0.68 *$ & $0.74 *$ \\
\hline VolCom & & & & & & & & & & - & $0.40^{*}$ \\
\hline CalFuste & & & & & & & & & & & - \\
\hline
\end{tabular}

Nota: DAP: Diámetro a la altura del pecho, AltCom: altura comercial, VolCom: volumen comercial, CalFuste: calidad del fuste.

${ }^{*}$ La significancia de la correlación corresponde $\mathrm{a} *=\mathrm{p}<0.05 ; * * \mathrm{p}<0.01$. 


\begin{tabular}{|c|c|c|c|c|c|c|}
\hline \multirow[b]{2}{*}{ Ranking } & \multicolumn{6}{|c|}{ Edad (meses) } \\
\hline & \multicolumn{2}{|c|}{10} & \multicolumn{2}{|c|}{22} & \multicolumn{2}{|c|}{34} \\
\hline 1 & 0,0137 & (34) & 0,1594 & (34) & 0,2515 & (17) \\
\hline 2 & 0,0133 & (38) & 0,1593 & (36) & 0,2475 & (34) \\
\hline 3 & 0,0130 & (60) & 0,1581 & (17) & 0,2451 & (38) \\
\hline 4 & 0,0129 & (59) & 0,1568 & (52) & 0,2436 & (36) \\
\hline 5 & 0,0128 & (51) & 0,1556 & (28) & 0,2427 & (52) \\
\hline 6 & 0,0128 & (17) & 0,1548 & (32) & 0,2420 & (45) \\
\hline 7 & 0,0127 & (41) & 0,1541 & (37) & 0,2413 & (47) \\
\hline 8 & 0,0126 & $(40)$ & 0,1536 & (50) & 0,2408 & (14) \\
\hline 9 & 0,0125 & (61) & 0,1530 & (21) & 0,2402 & (59) \\
\hline 10 & 0,0125 & (48) & 0,1526 & (58) & 0,2397 & (37) \\
\hline 11 & 0,0124 & (63) & 0,1522 & (39) & 0,2392 & (55) \\
\hline 12 & 0,0124 & (28) & 0,1517 & (10) & 0,2388 & (12) \\
\hline 13 & 0,0123 & (39) & 0,1513 & (47) & 0,2385 & (46) \\
\hline 14 & 0,0123 & (55) & 0,1510 & (38) & 0,2380 & (28) \\
\hline 15 & 0,0123 & (50) & 0,1507 & (63) & 0,2376 & (13) \\
\hline 16 & 0,0122 & (58) & 0,1504 & (14) & 0,2372 & (11) \\
\hline 17 & 0,0122 & (46) & 0,1501 & (20) & 0,2368 & (10) \\
\hline 18 & 0,0122 & (45) & 0,1498 & (22) & 0,2365 & (48) \\
\hline 19 & 0,0122 & (10) & 0,1495 & (12) & 0,2361 & (63) \\
\hline 20 & 0,0121 & (54) & 0,1493 & (48) & 0,2358 & (44) \\
\hline 21 & 0,0121 & (42) & 0,1490 & (54) & 0,2354 & (50) \\
\hline 22 & 0,0121 & (11) & 0,1488 & (25) & 0,2351 & (21) \\
\hline 23 & 0,0120 & (30) & 0,1486 & (45) & 0,2348 & (20) \\
\hline 24 & 0,0120 & (37) & 0,1483 & (13) & 0,2345 & (39) \\
\hline 25 & 0,0120 & (20) & 0,1481 & (44) & 0,2342 & (58) \\
\hline 26 & 0,0119 & (36) & 0,1479 & (55) & 0,2338 & (22) \\
\hline 27 & 0.0119 & (64) & 0,1476 & (24) & 0.2335 & (54) \\
\hline 28 & 0.0119 & (21) & 0,1474 & (16) & 0.2332 & (62) \\
\hline 29 & 0,0118 & (22) & 0,1472 & (26) & 0,2329 & (16) \\
\hline 30 & 0,0118 & (52) & 0,1469 & (59) & 0,2325 & (24) \\
\hline 31 & 0,0118 & (43) & 0,1467 & (62) & 0,2322 & (27) \\
\hline 32 & 0,0117 & (12) & 0,1465 & (9) & 0,2319 & (18) \\
\hline 33 & 0,0117 & (26) & 0,1462 & (29) & $\rightarrow 0,2315$ & (29) \\
\hline 34 & 0,0117 & (57) & 0,1460 & (27) & 0,2312 & (49) \\
\hline 35 & 0,0116 & (29) & 0,1457 & (15) & 0,2308 & (61) \\
\hline 36 & 0,0116 & (14) & 0,1455 & (46) & 0,2305 & (26) \\
\hline 37 & 0,0116 & (62) & 0,1452 & (11) & 0,2301 & (32) \\
\hline 38 & 0,0116 & (32) & 0,1450 & (51) & 0,2298 & (64) \\
\hline 39 & 0,0115 & (49) & 0,1447 & (61) & 0,2294 & (25) \\
\hline 40 & 0,0115 & (13) & 0,1444 & (18) & 0,2291 & (57) \\
\hline 41 & 0,0115 & (35) & 0,1442 & $(60)$ & 0,2287 & (33) \\
\hline 42 & 0,0115 & (47) & 0,1439 & (57) & 0,2284 & (15) \\
\hline 43 & 0,0114 & (24) & 0,1437 & (49) & 0,2281 & (31) \\
\hline 44 & 0,0114 & (33) & 0,1434 & (33) & 0,2277 & (30) \\
\hline 45 & 0,0114 & (15) & 0,1432 & (64) & 0,2274 & (41) \\
\hline 46 & 0,0113 & (44) & 0,1429 & (23) & 0,227 & (43) \\
\hline 47 & 0,0113 & (31) & 0,1426 & (31) & 0,2267 & (60) \\
\hline 48 & 0,0113 & (9) & 0,1423 & (42) & 0,2264 & (9) \\
\hline 49 & 0,0112 & (27) & 0,1420 & (41) & 0,226 & (23) \\
\hline 50 & 0,0112 & (23) & 0,1417 & (30) & 0,2257 & (42) \\
\hline 51 & 0,0112 & (18) & 0,1414 & (35) & 0.2253 & (51) \\
\hline 52 & 0,0111 & (16) & 0,1411 & (43) & 0,2248 & (40) \\
\hline 53 & 0,0111 & (25) & $\rightarrow 0,1407$ & (19) & ${ }^{-} 0,2243$ & (35) \\
\hline 54 & 0,0110 & (19) & 0,1403 & (40) & 0,2236 & (19) \\
\hline 55 & 0.0110 & (T) - & $\rightarrow 0.1398$ & (T) & $\rightarrow 0.2226$ & (T) \\
\hline
\end{tabular}

Figura 2. Ranking genético del volumen comercial de Gmelina arborea de una plantación de 10, 22 y 34 meses de edad, en Cariari de Pococí, provincia de Limón, Costa Rica. Nota: Los valores entre paréntesis corresponden al número de clon.

un DAP promedio de $11.76 \mathrm{~cm}$ y un volumen comercial de $0.063 \mathrm{~m}^{3}$ en 18 meses (Salas, 2012). Kumar (2007), reportó para Gmelina arborea un crecimiento en DAP y altura promedio a los 24 meses de edad, con valores de $7.2 \mathrm{~cm}$ y $5.18 \mathrm{~m}$, respectivamente, que, al compararlos con los resultados obtenidos a los 22 meses en este trabajo, reflejan diferencias inferiores al $50 \%$, mostrando el buen crecimiento de los genotipos evaluados.

El coeficiente de variación genético para los diferentes caracteres evaluados en este estudio, muestran que a la edad de 34 meses existe variabilidad genética importante, la cual se manifiesta en el material genético utilizado. Los clones con mejor desempeño en DAP, altura comercial, volumen comercial y calidad del fuste son el 17 y 34, superando la media del ensayo en todos los caracteres evaluados, caso contrario sucede con el clon 19 y el testigo, cuyos valores para las variables evaluadas son menores al resto del material evaluado. Lo anterior, aplica para las tres evaluaciones realizadas, donde se mantuvo dicho comportamiento, que gráficamente, se observa en la figura 1.

Parámetros genéticos: su estimación constituye una herramienta útil para la selección y la predicción de ganancias genéticas (Kumar, 2007). Para el caso específico de este trabajo, los conjuntos fueron evaluados a los 10, 22 y 34 meses de edad, tiempo relativamente reducido para la toma de decisiones relacionadas con la selección genética. En general, todos los caracteres presentaron bajos coeficientes de heredabilidad individual, lo cual puede tener explicación con la edad de la plantación, en la cual el material evaluado aún no ha expresado su potencial genético (Ávila et ál., 2015; Pastrana-Vargas, Espítia-Camacho, \& Murillo-Gamboa, 2012).

La calidad del fuste fue el carácter que mostró mayores valores de heredabilidad individual y media del clon, así como un alto coeficiente de valor relativo, lo que permite considerar esta variable como un posible parámetro de selección, siempre que se contemple la influencia de factores externos, 
principalmente, el manejo silvicultural, como podas, raleos, daños por viento, entre otros, los cuales pueden afectar la valoración de la calidad del fuste y mostrar diferencias a la hora de cuantificar dicha variable en las plantaciones forestales de G. arborea.

Un factor para considerar a la hora de analizar los resultados obtenidos de heredabilidad individual en sentido amplio, es el valor del coeficiente de variación ambiental, que aumenta sistemáticamente, conforme la edad, lo que explica en parte los resultados obtenidos para el parámetro de heredabilidad individual.

La heredabilidad media del clon a los 34 meses de edad muestra valores superiores a 0.70 para los caracteres evaluados, donde el DAP, el volumen comercial y la calidad del fuste presentaron los mayores valores, con una precisión superior a 0.88 , lo que permite mayor confiabilidad en caso de seleccionar con base en dichos caracteres (Pastrana-Vargas et al., 2012).

De acuerdo con Resende (2002), la precisión se clasifica como: muy alta ( $>$ $0.90)$, alta (0.70 a 0.90), moderada (0.50 a 0.70$)$ y baja $(<0.50)$. Para los caracteres evaluados los valores se clasifican como altos, que de acuerdo con Resende (1995), en cuanto mayor sea la precisión mayor será la exactitud de la selección y, por lo tanto, mayor será la ganancia genética.

El coeficiente de variación genético permite conocer la variabilidad existente dentro del material genético (Resende, 2007), el cual, para este estudio, se obtuvo un descenso en este parámetro conforme aumenta la edad, probablemente, debido a un aumento en el coeficiente de variación ambiental, a excepción de la calidad del fuste, donde se reflejó un comportamiento diferente con relación en los demás caracteres, mostrando una mayor variabilidad.
Los resultados para el valor genético del volumen comercial, obtenido a la edad de 34 meses y que se muestran en la figura 1 , donde la distribución de los clones con relación en su valor genético es muy regular, no presentan un rango muy amplio entre los límites de dichos valores genéticos, de ahí que a pesar de existir un grupo de clones que sobrepasan la media, la diferencia no es muy significativa.

Correlaciones genéticas: estas permiten determinar qué tan estable se mantiene el ranking genético, por lo tanto, entender la asociación de diversos rasgos con el crecimiento de G. arborea, también, posibilitan realizar selección genética a una edad determinada, con mayor exactitud y precisión. En el caso de este estudio, se muestran correlaciones altas y positivas entre los caracteres DAP- volumen comercial y entre la altura comercial - calidad del fuste.

Además, las mayores correlaciones se muestran a los 34 meses de edad, donde el DAP y el volumen comercial presenta una correlación estadísticamente significativa $(\mathrm{r}=0.97 ; \mathrm{P}<0.01)$, mientras que la altura comercial y el carácter calidad del fuste reflejan un valor de $0.74(\mathrm{P}<0.05)$ de correlación genética, valores superiores a 0.80 se obtuvieron cuando se correlaciona el DAP consigo mismo, también, a los 34 meses. Estos valores de correlación indican que una selección a partir de estos rasgos puede proporcionar avances importantes en los programas de mejoramiento genético con G. arborea.

Las bajas correlaciones obtenidas o incluso negativas entre algunos caracteres muestran la poca asociación entre esos rasgos, donde el aumento de un carácter conduce a la disminución del otro, de manera que las posibilidades de selección a partir de dichas variables no son factibles. La 
correlación entre las variables cuantitativas con la calidad del fuste, fueron en su mayoría muy bajas o incluso algunas negativas, a excepción de altura comercial, que correlaciono positivamente, con la calidad, a los 22 y 34 meses de edad, por lo general, la tendencia es a obtener altas correlaciones entre las variables cuantitativas referidas a una misma edad (Pavlosky y Murillo, 2013).

El comportamiento obtenido en las correlaciones genéticas entre los caracteres, permite establecer que la edad de 34 meses es la óptima para realizar selección, fundamentado, también, en los valores de heredabilidad; sin embargo, es importante tener presente que entre mayor edad los coeficientes de los parámetros genéticos obtenidos pueden incrementar, suponiendo una mayor expresión genética del material clonal utilizado.

Ranking genético: las diferencias entre clones se observan con mayor claridad a la hora de observar este ranking, referido a cualquiera de los caracteres evaluados. La figura 1 muestra su distribución de acuerdo con el carácter volumen comercial, a diferentes edades (10, 22 y 34 meses). El comportamiento en el nivel de clones a lo largo del período de evaluación permite conocer la tendencia de algunos individuos a subir y otros a descender, lo anterior, producto de la expresión genética que van mostrando conforme aumenta la edad, donde algunos manifiestan superioridad genética y otros que por el contrario tienen a decaer.

Al dividir el Ranking en grupos de clones de interés, de acuerdo con su posición, se diferencian un primer grupo, el cual está conformado por aquellos individuos que muestran un comportamiento superior a los demás, en los cuales algunos se mantienen desde los 10 meses de edad hasta los 34 meses, como es el caso del clon 34, seguido por el 17, otros se mantienen en los primeros lugares a partir de los 22 meses, como el 36 y el 52. Los anteriores, constituyen el grupo con los cuales se garantiza una mayor ganancia genética, a la hora de hacer selección.

Otro grupo compuesto por clones que ocupan una posición intermedia, 10, 22, 39 , 50, entre otros se muestran como opción para una potencial selección genética, donde el porcentaje de ganancia es menor al primer grupo, sin embargo, siempre se obtienen resultados mayores al promedio general. El tercer grupo está constituido por aquellos individuos que siempre estuvieron dentro las últimas posiciones en el ranking genético, cuya ganancia será menor comparada con los demás los del primero y segundo grupo; dentro de esta clasificación se encuentran el testigo, que siempre estuvo en la última posición, durante todo el período de evaluación, el clon 19, 40, 35, entre otros.

La utilidad práctica del ranking genético, además de que permite la proyección de potenciales ganancias genéticas, es que suele ser un indicador de cuándo realizar evaluaciones más prolongadas en el tiempo, con el objetivo de verificar cuáles son los clones elite, que se comportan con clara superioridad y de esta forma apoyar con mayor seguridad los programas de mejoramiento genético de la especie. Así como también, permite conocer clones de rápido, mediano y lento crecimiento, que permitan direccionar los objetivos de producción forestal (Kumar et ál., 2010).

\section{CONCLUSIONES}

Los parámetros genéticos obtenidos para Gmelina arborea muestran valores de heredabilidad media del clon, altos para las variables estudiadas, especialmente, la calidad del fuste, que presentó una superior 
0.8, a partir de los 22 meses de edad, lo que permiten suponer el potencial que tienen los programas de mejoramiento genético de la especie. Existen clones estables, que garantizan una mayor ganancia genética tanto en DAP, como en volumen comercial, específicamente, los 17, 34, son los que mejor se ubican en el ranking, a diferencia de los 19, $40 \mathrm{y}$ el testigo, que se ubicaron en las últimas posiciones.

La estimación de los parámetros genéticos obtenidos, principalmente en DAP, volumen comercial y calidad del fuste, mostraron los mayores coeficientes dichos caracteres, además se presentan correlaciones altas y positivas entre el DAP y el volumen comercial, durante el período de evaluación, posibilitando la selección indirecta a partir de los 10 meses y hasta los 34 meses, definiendo dicho rango de edad como posible edad de selección. Evaluaciones posteriores a los 34 meses permiten validar los resultados obtenidos y conocer potenciales cambios en el ranking genético.

De acuerdo con los resultados obtenidos podría esperarse que las variables DAP, volumen comercial y la calidad del fuste reflejen un mayor control genético, al presentar los valores más altos de los distintos parámetros genéticos evaluados.

\section{RECONOCIMIENTOS}

A la Universidad Nacional de Costa Rica y al Consejo Nacional para Investigaciones Científicas y Tecnológicas por el soporte económico para la realización de la investigación, así como también, a la Cooperativa de Mejoramiento Genético Forestal (GENFORES), por el apoyo en la logística de las actividades de campo para el levantamiento de la información.

\section{DECLARACIÓN DE LA CONTRIBUCIÓN DE LOS AUTORES}

El porcentaje total de contribución para la conceptualización, preparación y corrección de este artículo fue el siguiente: W.H.C $40 \%$, O.M.G.30 \% y Y.B.V. $30 \%$.

\section{DECLARACIÓN DE DISPO- NIBILIDAD DE LOS DATOS}

Los datos que respaldan los resultados de este estudio serán puestos a disposición por el autor correspondiente [W. H. C.], previa solicitud razonable.

\section{REFERENCIAS}

Ávila-Arias, C., Murillo-Cruz, R., Murillo-Gamboa, O., \& Sandoval-Sandoval, C. (2015). Desarrollo juvenil de clones de Gmelina arborea Roxb. De dos procedencias, en sitios planos del Pacífico Sur de Costa Rica. Revista Forestal Mesoamericana Kurú, 12(28), 23-25. https://doi.org/10.18845/rfmk.v12i28.2097

Badilla, Y. \& Murillo, O. (2011). Avances en el mejoramiento genético de la teca en GENFORES, Costa Rica. En: Conferencia Forestal Internacional: Bosques plantados de teca. San José, Costa Rica.

Balcorta, H. y Vargas, J. (2004). Variación fenotípica y selección de árboles en una plantación de melina (Gmelina arbórea Linn., Roxb.) de tres años de edad. Revista Chapingo, 10(1), 13-19.

Barrantes, A. R. \& Ugalde, S. A. (2019). Usos y aportes de la madera en Costa Rica. Oficina Nacional Forestal. https://onfcr.org/ usos-y-aportes-de-la-madera-en-costa-rica/

Chavarría, E. M. I. (2016). Reporte Estadístico Forestal 2014-2015. Sistema Nacional de Áreas de Conservación. San José, Costa Rica.

Cruz, C. D. (2005). Princípios de genética quantitativa. Viçosa, MG: UFV.

Falconer, D. S. (1987). Introdução a genética quantitativa. Viçosa, MG: UFV. 
Foster, G. S., \& Shaw, D. V. (1988). Using clonal replicates to explore genetic variation in a perennial plant species. Theoretical and Applied Genetics, 76(5), 788-794. https://doi. org/10.1007/BF00303527

Instituto Meteorológico Nacional [IMN]. (2005). Atlas Climatológico de Costa Rica. Ministerio del Ambiente y Energía, San José, Costa Rica. Disponible en: https://www.imn.ac.cr/ atlas-climatologico.

Instituto Nacional de Estadística y Censos [INEC]. (2014). VI Censo Nacional Agropecuario. INEC, Instituto Nacional de Estadística y Censos. San José, Costa Rica.

Kumar, A. (2007). Growth Performance and Variability in Different Clones of Gmelina arborea (Roxb.). Silvae Genetica, 56(1-6), 3236. https://doi.org/10.1515/sg-2007-0005

Kumar, A.; Luna, R. K. \& Parveen, K. V. (2010). Variability in growth characteristics for different genotypes of Eucalyptus tereticornis (SM.). Journal of Forestry Research. 21(4), 87-491. https://doi.org/10.1007/s11676-010-0103-2

Marques-Júnior, O. G. (1995). Estimativas dos parâmetros genéticos e fenotípicos e avaliação da eficiência da seleção precoce em Eucalyptus cloeziana F. Muell. (Teses de mestrado inédita), Universidade Federal de Lavras, Lavras, MG. http://repositorio.ufla.br/handle/1/36888

Mata-Chinchilla, R., \& Castro-Chinchilla, J. (2019). Geoportal de suelos de Costa Rica como Bien Público al servicio del país. Revista Tecnología en Marcha, 32, 51-56. https://doi. org/10.18845/tm.v32i7.4259

Moya, R. (2004). Gmelina arborea en Costa Rica. Bois et Forest Des Tropiques, 279(1). 47-57. https:// www.researchgate.net/publication/276277090 Gmelina_arborea_in_Costa_Rica

Murillo, O. \& Guevara, V. (2013). Estado de los recursos genéticos forestales de Costa Rica. MINAET/FAO/CONAGEBIO, San José, Costa Rica.

Murillo, O. (1992). Diseño de un huerto semillero de Gmelina arborea para la producción de semilla certificada en la zona norte de Costa Rica. Tecnología en Marcha, 11(3), 51-58.

Murillo, O., Meza, A., \& Cabrera, J. M. (2004). Estimación del valor real y del valor de mercado en pie de la plantación forestal. Revista Agronomía Costarricense, 28(1), 47-55. http:// www.redalyc.org/articulo.oa?id=43628105
Pastrana-Vargas, I., Espítia-Camacho, M., \& Murillo-Gamboa, O. (2012). Evaluación del potencial de mejoramiento genético en el crecimiento en altura de Acacia mangium Willd. Acta Agronómica, 61(2), 143-150. http:// www.scielo.org.co/scielo

Pavlotzky, B. \& Murillo, O. (2013). Ganancia genética esperada en Acacia mangium en San Carlos, Zona Norte de Costa Rica. Revista Forestal Mesoamericana Kurú, 10(24), 14. https:// doi.org/10.18845/rfmk.v10i24.1320

Resende, M. D. V. (2007). SELEGEN-REML/BLUP: Sistema estatístico e seleção genética computadorizada via modelos lineares mistos. Embrapa Informação Tecnológica, Colombo: Embrapa Florestas.

Resende, M. D. V. de. (2002). Genética biométrica e estatística no melhoramento de plantas perenes. Embrapa Informação Tecnológica, Colombo: Embrapa Florestas.

Resende, M. D. V. (1995). Delineamento de experimentos de seleção para maximização da acurácia seletiva e do progresso genético. Revista Árvore, 4(19), 479-500.

Salas, R. A. (2012). Evaluación de un ensayo genético de Gmelina arborea en Siquirres, Limón (Tesis de licenciatura inédita). Instituto Tecnológico de Costa Rica, Cartago, Costa Rica. https://repositoriotec.tec.ac.cr/ handle/2238/2949

Vencovsky, R. \& Barriga, P. (1992). Genética Biométrica no Fitomelhoramento. Ribeirão Preto: Sociedade Brasileira de Genética.

Zeaser, D. (1998). Programa de mejoramiento genético de la Ston Forestal en la zona sur de Costa Rica. En Seminario Aumento de la rentabilidad de las plantaciones forestales: un reto ligado al uso de semilla de alta calidad. San José, Costa Rica. 19 de mayo de 1998. Memorias de CD-ROM. San José, Costa Rica.

Zimback, L.; Mori, E. S.; Brizolla, T. F. \& Chaves, R. (2011). Correlações entre caracteres silviculturais durante o crescimento de Eucalyptus grandis Hill ex Maiden. Revista do Instituto Florestal, 23(1), 57-67. http://iflorestal.sp.gov.br/publicacoes-if/revista-do-if/ sumario_v23_1/

Zobel, B. \& Talbert, J. (1984). Applied forest tree improvement. New York: John Wiley \& Sons. 


\section{(c) (1) ()$_{\mathrm{BY}} \mathrm{NC}$}

Estimación de parámetros genéticos de Gmelina arborea Roxb. (melina) en el Caribe de Costa Rica (William Hernández-Castro • Yorleny Badilla Valverde • Olman Murillo-Gamboa) Uniciencia is protected by Attribution-NonCommercial-NoDerivs 3.0 Unported (CC BY-NC-ND 3.0) 